Laser Chem., Vol. 16, pp. 219-228

Reprints available directly from the publisher Photocopying permitted by license only
(C) 1996 OPA (Overseas Publishers Association) Amsterdam B.V. Published in The Netherlands by Harwood Academic Publishers GmbH Printed in Malaysia

\title{
A NEW PERSISTENT PHOTON-GATED SPECTRAL HOLE-BURNING SYSTEM: ZINC TETRAPHENYLPORPHYRIN LINKED TO POLYVINYL- BENZYLCHLORIDE-CO-POLYSTYRENE
}

\author{
S. SALHI ${ }^{+}$, S. KULIKOV ${ }^{++}$, C. BIED-CHARRETON and J. P. GALAUP \\ Laboratoire de Photophysique et Photochimie Supramoléculaires \\ et Macromoléculaires (URA 1906 du CNRS) \\ Ecole Normale Supérieure de Cachan \\ 61, Avenue du Président Wilson, 94235 CACHAN Cedex (France)
}

(Received 5 July, 1995)

\begin{abstract}
Two colour photon-gated persistent spectral hole-burning via donor-acceptor electron transfer is reported in systems where the donor: zinc tetraphenylporphyrin (ZnTPP) and the acceptor: vinylbenzylchloride where linked to a polystyrene chain. A spectral hole is observed when the sample is irradiated using a gating light in addition to the wavelength-selective light. No effect of the gating light has been observed for ZnTPP dispersed in polystyrene (PS) even with efficient acceptors. In contrast, the efficiency of hole formation under two colour excitation is four times larger than that under one colour excitation in ZnTPP-PBCS/PMMA and nearly two times larger in ZnTPP-PBCS/PS.
\end{abstract}

KEY WORDS: Hole-burning, photon-gating, electron transfer, zinc tetraphenylporphyrin, grafted molecules, copolymer.

\section{INTRODUCTION}

Persistent Spectral Hole-Burning (PSHB) is a phenomenon in which a stable hole is formed in the inhomogenously broadened absorption band of impurity centers at very low temperature by selective excitation with a narrow band laser. PSHB has attracted much interest as a very sensitive probe for the dynamics in polymer systems as well as a possible means for high density optical data storage. ${ }^{1}$ While the first years of PSHB research were focused on single photon mechanisms, ${ }^{2}$ the destructive reading that occurs in these systems during hole detection has generated a great interest for two colour photon-gated materials. In photon-gating PSHB, two photons are required for the photoinduced change leading to a higher hole-burning

\footnotetext{
${ }^{+}$Present address : Faculté des Sciences, Université Mohammed 1 ${ }^{\text {er }}$, Oujda (Maroc).

${ }^{++}$Permanent address: Institute of Spectroscopy, Russian Academy of Sciences, Troitzk, 142092 Moscow region (Russia).
} 
efficiency; nevertheless, only one wavelength is required to probe the unreacted ground state population during hole detection. With such a mechanism, the two stages of the PSHB procedure (writing the spectral hole first, then reading it) can be well separated.

The various photon gated PSHB systems that have been reported as yet can be divided into four types, based on the kind of hole formation photoreactions that are involved, namely, ionisation, ${ }^{3-5}$ decomposition, ${ }^{6-8}$ Donor-Acceptor Electron Transfer (DA-ET) ${ }^{9,10}$ and sensitisation. ${ }^{11,12}$

In almost all the reported photon-gated PSHB systems involving DA-ET photoreactions, porphyrin derivatives are used as donor chromophores and halogenated compounds as acceptors. ${ }^{13,14}$ In these systems, an acceptor must be located in the neighbourhood of the donor porphyrin for a high efficiency. Then, the matrix should contain the acceptor in a high concentration. To achieve this, a polymer which has the electron acceptor directly bonded to the polymer chain has been used. ${ }^{14,15}$ The branching of the guest molecule to the host matrix is a very effective way to make highly doped polymer materials without aggregation of the guest molecules. Previous studies have demonstrated that the grafting of donors and/or acceptors to the host matrix can solve such problems as low burning efficiencies and low thermal stability of burnt holes in PSHB materials via DA-ET photoreactions. ${ }^{13}$ It is also expected that the grafting of the chromophore to the host should reduce the influence of spectral diffusion at low temperature. These characteristics are important for the application of PSHB materials to frequency domain optical data storage devices or optical data processing.

In this paper, we report on two colour photon-gated PSHB via a DA-ET reaction in which a polystyrene chain contains a vinylbenzylchloride in its monomer repeated unit as acceptor molecules and grafted ZnTPP as donor molecules. Two polymers: polystyrene (PS) and polymethylmethacrylate (PMMA) were used as hosts. The hole formation efficiency of ZnTPP linked to polyvinylbenzylchloride-copolystyrene which we call ZnTPP-PBCS hereafter, has been compared to the values obtained with ZnTPP dispersed in PS or in PMMA.

\section{EXPERIMENTAL}

The chemical structure of the compound studied in this letter is shown in Figure 1. ZnTPP-PBCS has been synthesised by grafting ZnTPP to a preformed polystyrene. We used the Williamson reaction starting from polyvinylbenzylchloride-co-polystyrene and porphyrin monofunctionalized by a phenol substituent as reported previously. ${ }^{16}$ The amount of grafted porphyrins was evaluated to be: 1 porphyrin for 3 benzylchloride. $\mathrm{ZnTPP}$ and $\mathrm{ZnTPP}-\mathrm{PBCS}$ were dispersed in PS (MW $\approx 430000)$ and PMMA (MW $\approx 120000$ ) by dissolving them in toluene. Sample films were prepared by a film casting method. The mixture was dried over one week. The concentration of porphyrins in the matrices $\left(\approx 10^{-4} \mathrm{~mol} / \mathrm{l}\right)$ was adjusted to give thin slabs $(\mathrm{e} \approx 1 \mathrm{~mm})$ with an absorbance value at the burning wavelength $\left(\lambda_{1}\right)$ of about 0.5 at room temperature.

The samples were placed in a liquid helium cryostat with variable temperature facilities (SMC - l'Air Liquide). They were irradiated at $5 \mathrm{~K}$ simultaneously with 


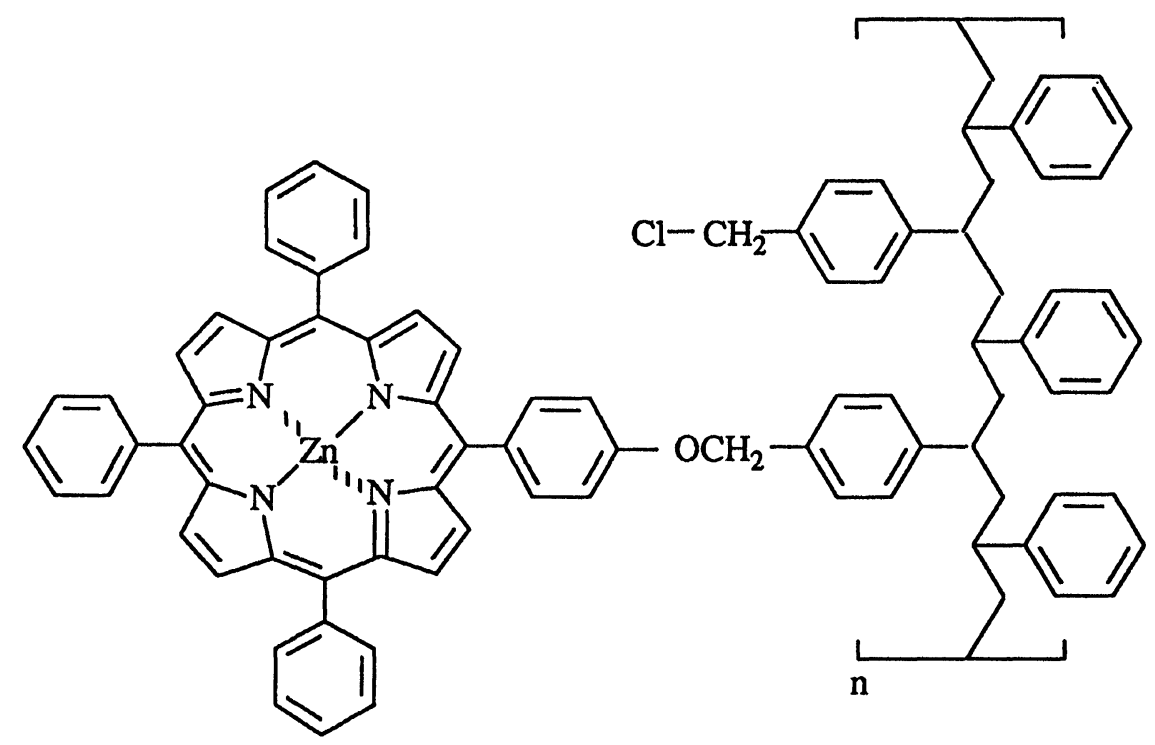

Figure 1 Chemical structure of the synthesized ZnTPP-PBCS. The average distribution along the polymer chain is as follows: 1 ZnTPP for 3 benzylchloride and 18 styrene.

selective laser light from a Rhodamine $6 \mathrm{G}$ ring dye laser $\left(\lambda_{1}\right.$ tunable around $\left.600 \mathrm{~nm}\right)$ and with the gating light from an argon laser (Spectra Physics, Model 2016) used in all-lines regime ( $\lambda_{2}$ chosen among the two lines at 488 and $514 \mathrm{~nm}$ ). For burning, the two beams, non focused, were overlapped in the same spatial spot on the sample. The intensities of both beams were adjusted by using a combination of neutral density filters. To record the holes, we monitored the fluorescence intensity through a low-pass colour filter when scanning the dye laser wavelength. The signals were processed with a lock-in amplifier and fed into a microcomputer for data acquisition and treatment. Holes were burnt with an excitation intensity not greater than 1.4 $\mathrm{mW}$ on a spot area of $0.1 \mathrm{~cm}^{2}$ on the sample, corresponding to power densities of about $14 \mathrm{~mW} / \mathrm{cm}^{2}$. The hole area was kept constant for all the experiments. The readout of the holes was performed with a beam attenuated by a factor of about $10^{3}$. Burning times were varied around a few minutes depending on the fluence required to produce a detectable hole.

The holes were fitted by Lorentzians and the values of width and area were obtained from them.

\section{RESULTS AND DISCUSSION}

The absorption spectrum of ZnTPP-PBCS measured with an UV-visible spectrometer (Philips PU8700) is shown in Figure 2. This absorption spectrum is almost the same as for dilute $\mathrm{ZnTPP}$ in solution. Therefore, we conclude that the covalent 


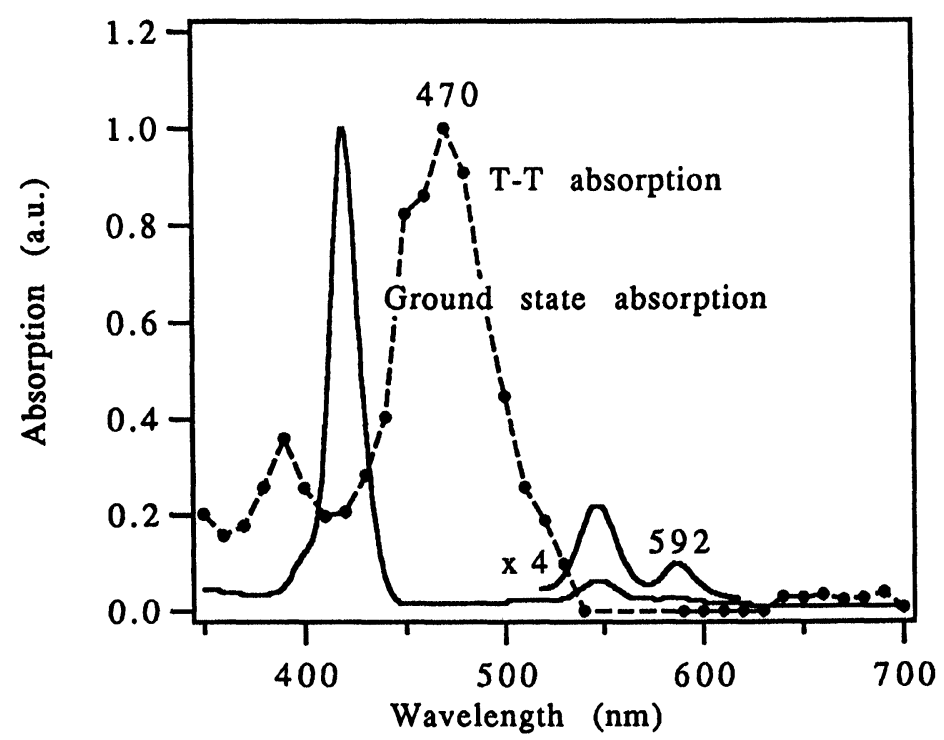

Figure 2 Ground state absorption of ZnTPP-PBCS (full line) and triplet-triplet absorption spectra of ZnTPP (dashed line) in a toluene solution.

bonds introduced between ZnTPP and polyvinylbenzylchloride-co-polystyrene do not have a large influence on the electronic states of the $\pi$ conjugated system of the zinc porphyrin ring. We measured the triplet-triplet absorption of ZnTPP using the flash photolysis method on a nitrogen-bubbled toluene solution. The result is drawn in Figure 2. The maximum of the triplet-triplet absorption appears at $470 \mathrm{~nm}$. As the inhomogeneous absorption band of the $\mathrm{Q}(0,0) \mathrm{S}_{1} \leftarrow \mathrm{S}_{0}$ transition has its maximum around $\approx 592 \mathrm{~nm}$, we used dye laser wavelengths between $570 \mathrm{~nm}$ and $620 \mathrm{~nm}$ for the site selection, and argon laser lines at 488 and $514 \mathrm{~nm}$ for the gating light. $\mathrm{ZnTPP}$, excited to the $\mathrm{S}_{1}$ state, undergoes intersystem crossing with a very high efficiency $(\varphi \approx 0.90)^{17}$ to the triplet state $T_{1}$. Due to the long lifetime of the $T_{1}$ state, ZnTPP can then be further excited to a higher triplet state $T_{n}$ by the absorption of a photon of the gating light $\left(\lambda_{2}\right)$. ZnTPP is favorable because the wavelengths of $\lambda_{2}$ at 488 and $514 \mathrm{~nm}$ are quite close to the absorption maximum of the T-T transition and because its ground state only has a weak absorption cross section in the $\lambda_{2}$ region.

We investigated the dependence of the hole characteristics in polymer matrices using the same guest concentration. In the case of PMMA, easily detectable holes were observed after a few minutes of irradiation. Much longer times were necessary to burn holes in the PS matrix.

A persistent spectral hole burnt in ZnTPP-PBCS/PMMA is shown in Figure 3. In trace (a), the hole was burnt via a one color excitation: the sample was irradiated with only a frequency selective excitation $\left(\lambda_{1}=605.8 \mathrm{~nm}\right.$, duration $=10 \mathrm{mn}$, burning intensity $=1.3 \mathrm{~mW}$ ). In trace (b), the persistent spectral hole was burnt via a twocolor excitation: the sample was simultaneously irradiated with a frequency selective 


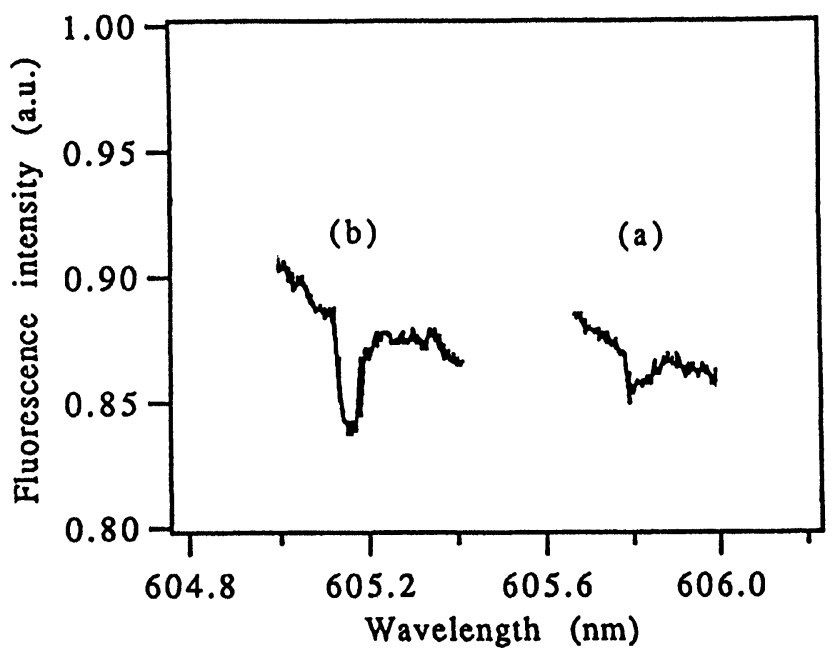

Figure 3 One and two photon holes burnt in a sample of ZnTPP-PBCS/PMMA at 5 K, burning time $=10 \mathrm{mn}, \mathrm{P} \lambda_{1}=1.3 \mathrm{~mW}$ : (a) one colour, $\lambda_{1}=605.8 \mathrm{~nm}$ : (b) two colour, $\lambda_{1}=605.2 \mathrm{~nm}, \lambda_{2}=488 \mathrm{~nm}$, $\mathrm{P} \lambda_{2}=7.5 \mathrm{~mW}$.

excitation $\left(\lambda_{1}=605.2 \mathrm{~nm}\right.$, duration $=10 \mathrm{mn}$, burning intensity $\left.=1.3 \mathrm{~mW}\right)$ and the gating excitation $\left(\lambda_{2}=488 \mathrm{~nm}\right.$, duration $10 \mathrm{mn}$, gating intensity $\left.=7.5 \mathrm{~mW}\right)$. The efficiency of hole formation under two colour excitation is four times larger than that under one colour excitation in ZnTPP-PBCS/PMMA. In contrast, no detectable influence of the gating light could be observed on the free dispersed ZnTPP/PMMA system without electron acceptors when irradiated under the same conditions.

The photon-gated hole spectrum shown in Figure 4 was obtained with the ZnTPP-PBCS/PS system, irradiated at $\lambda_{1}=603.0 \mathrm{~nm}$ using a burning laser intensity $=1.3 \mathrm{~mW}$ during $30 \mathrm{mn}$ for the one colour hole (trace $(\mathrm{a})$ ), and at $\lambda_{1}=601.8 \mathrm{~nm}$, duration $=30 \mathrm{mn}$, burning intensity $=1.3 \mathrm{~mW}$ with $\lambda_{2}=488 \mathrm{~nm}$, gating intensity $=8.0 \mathrm{~mW}$ for the two colour hole (trace (b)). The burning efficiency due to the gating light is increased almost two times in ZnTPP-PBCS/PS, compared to its value under one colour excitation. Again, no detectable influence of the gating light could be produced when a ZnTPP/PS sample was irradiated in the same conditions.

The mechanism of photon-gated hole formation for the ZnTPP-PBCS in PMMA or PS matrices can be understood as a laser induced donor-acceptor electron transfer. The presence of an halogen atom (chlorine) in the acceptor molecules (vinylbenzylchloride) is crucial for the persistence of spectral holes, because as just mentioned above, no gating effect could be obtained when ZnTPP is only dispersed in PMMA or in PS without any acceptor, under the same irradiation conditions as with the ZnTPP-PBCS systems.

The feasibility of a photoinduced electron transfer reaction for a D-A combination is dictated by the change in the free energy, $\Delta \mathrm{G}$, accompanying the reaction. ${ }^{18-20}$ An important requirement is the condition of the exothermicity $(\Delta \mathrm{G}<0)$ for the electron transfer reaction. $\Delta \mathrm{G}$ is calculated by the following equation [20] 


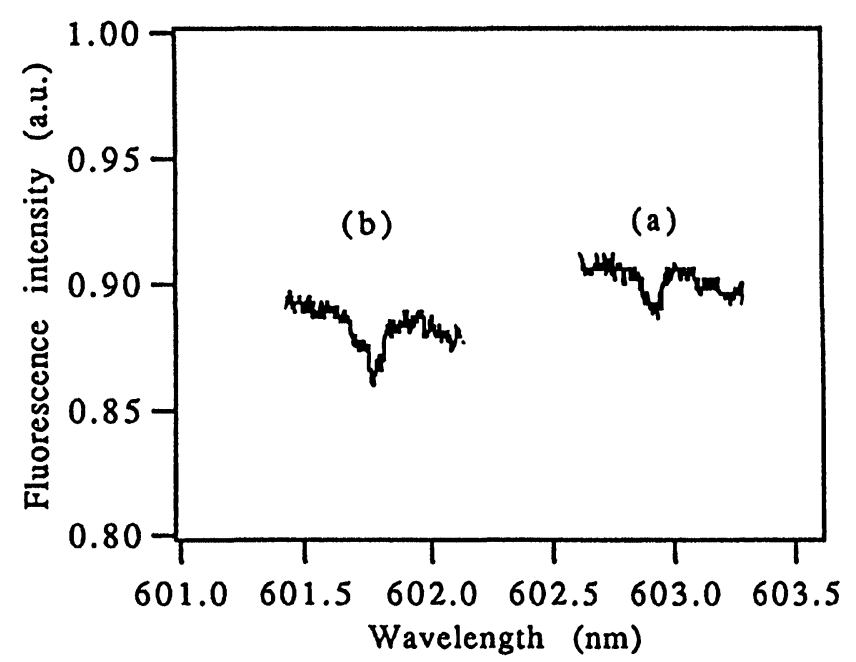

Figure 4 One and two photon holes burnt in a sample of ZnTPP-PBCS/PS at $5 \mathrm{~K}$, burning time $=30 \mathrm{mn}, \mathrm{P} \lambda_{1}=1.3 \mathrm{~mW}$ : (a) one colour, $\lambda_{1}=603.0 \mathrm{~nm}$; (b) two colour, $\lambda_{1}=601.8 \mathrm{~nm}, \lambda_{2}=488 \mathrm{~nm}$, $\mathrm{P} \lambda_{2}=8 \mathrm{~mW}$.

and has been applied by several researchers to photon-gated hole-burning ${ }^{9,21}$ :

$$
\Delta \mathrm{G}=\mathrm{E}_{\mathrm{ox}}-\mathrm{E}_{\mathrm{red}}-\mathrm{E}_{\mathrm{ex}}-\mathrm{e}^{2} /\left(\mathrm{R}_{\mathrm{c}} \cdot \varepsilon\right)
$$

where $E_{o x}$ is the one electron oxidation potential of an electron donor, $E_{r e d}$ is the one electron reduction potential of an electron acceptor and $\mathrm{E}_{\mathrm{ex}}$ is the excitation energy required to produce the reaction state. The last term: $-\mathrm{e}^{2} /\left(\mathrm{R}_{\mathrm{c}} \cdot \varepsilon\right)$ is the coulombic term which accounts for the electrostatic interaction between an ionic pair separated by the distance $R_{c}$. Compared to the values of the red-ox potentials, this term can be neglected.

For $\mathrm{ZnTPP}, \mathrm{E}_{\mathrm{ox}}=0.71 \mathrm{eV}$ versus $\mathrm{SCE},{ }^{22}$ for benzylchloride, $\Phi_{\mathrm{CH}} \mathrm{Cl}$, $\mathrm{E}_{\mathrm{red}}=-1.94 \mathrm{eV}$ versus SCE. ${ }^{23}$ The $\mathrm{E}_{\mathrm{ex}}$ value is evaluated as the sum of the lowest triplet energy $\left(1.59 \mathrm{eV}^{22}\right)$ and of the energy gap corresponding to the gating light at $488 \mathrm{~nm}(2.54 \mathrm{eV})$.

Therefore, in these conditions, the $\Delta G$ change is negative and equals to $-1.48 \mathrm{eV}$. Negative values for $\Delta \mathrm{G}$ are obtained with other electron acceptor molecules, as for instance the $\mathrm{p}$-hydroxybenzaldehyde ( $\mathrm{p}-\mathrm{HBA}, \mathrm{E}_{\mathrm{red}}=-1.68 \mathrm{eV},{ }^{24}$ which gives $\Delta \mathrm{G}=-1.74 \mathrm{eV}$ ). These thermodynamic arguments suggest that the photongated hole burning should be quite efficient with ZnTPP and a suitable electron acceptor in any kind of matrices.

Therefore, for comparison, we have investigated two photon hole-burning in samples of ZnTPP dispersed in PS or in PMMA with various acceptors. Unambiguously, the results obtained show that efficient two photon hole-burning is possible only in a PMMA host and no effect could be detected in PS. In a ZnTPP/pHBA/PS sample, we even observed that an intensive exposure of the sample to only 
the $\mathrm{Ar}^{+}$laser beam led to hole erasing. Figure 5 depicts the effect with several holes burnt successively at $593.8 \mathrm{~nm}, 594.4 \mathrm{~nm}$ and $595.1 \mathrm{~nm}$ for $10 \mathrm{mn}$ each. The intensity for burning was $5 \mathrm{~mW}$ and that of the gating light was about $18 \mathrm{~mW}$. As shown, no effect of the gating light is observed even with the presence of an efficient electron acceptor.

This result points out the importance of the host. In fact, there are only a few reports on the quantitative relation between hole-burning efficiency and matrix properties. In most cases, PMMA is used as the suitable amorphous matrix. ${ }^{9,14,25}$ Suzuki and al. showed that the hole formation yield increased from a PMMA to a polyethylene (PE) host matrix, depending on the donor-acceptor average separation. They discussed their result assuming the guest molecules are mainly located in the amorphous parts of the semicrystalline PE matrix, giving rise to a D-A average separation in PE shorter than in complete amorphous PMMA. ${ }^{26}$

In our case, we understand the negative result in the PS host as a consequence of the non-polar character of polystyrene. Due to the weakness of the polarity of the PS host, the electron transfer can not be stabilized, even at the liquid helium temperature. This means that the reverse reaction is so effective and fast that the photon-gated hole is refilled at once. A different situation exists in the polar host PMMA. The polarity of the matrix then allows a stabilisation of the charge redistribution. The important result demonstrated in this work is the fact that it was possible to by pass the barrier imposed by the polystyrene matrix with the ZnTPPPBCS/PS system where both the donor and the acceptor molecules are linked to the same chain. We believe that it is due to the high concentration of acceptor sites achieved in this new system, where the flexibility of the polymer chain close to the attached donor and acceptor moieties, as shown in Figure 6, allows an efficient

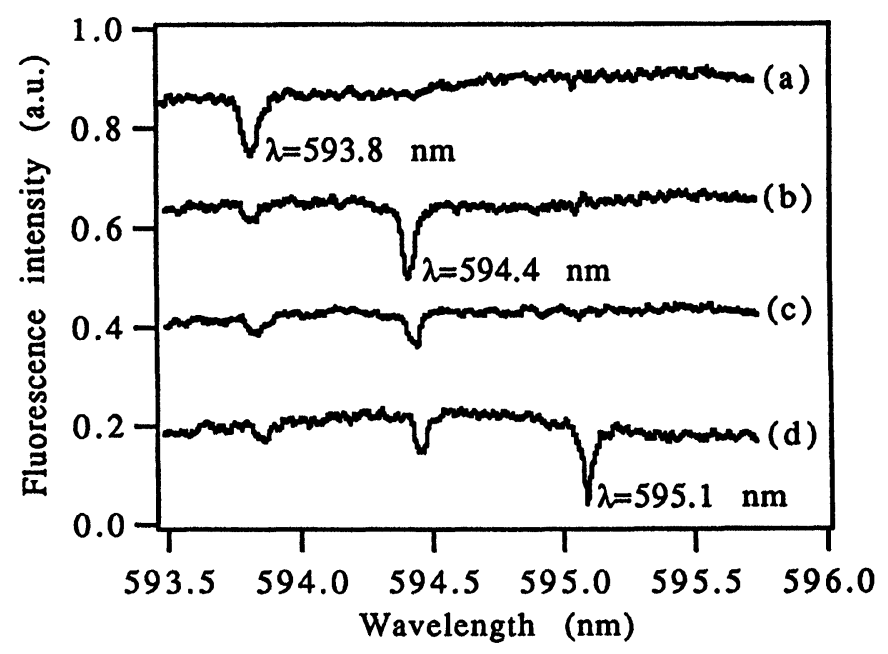

Figure 5 One and two photon holes burnt successively in a sample of ZnTPP/p-HBA/PS at $1.8 \mathrm{~K}$, burning time $=10 \mathrm{mn}, \mathrm{P} \lambda_{1}=5 \mathrm{~mW}, \lambda_{2}=488 \mathrm{~nm}, \mathrm{P} \lambda_{2}=18 \mathrm{~mW}$ : (a) two colour, $\lambda_{1}=593.8 \mathrm{~nm}, \lambda_{2}$; (b) two colour, $\lambda_{1}=594.4 \mathrm{~nm}, \lambda_{2} ;$ (c) only $\mathrm{Ar}^{+}$laser, $\lambda_{2} ;(d)$ only dye laser, $\lambda_{1}=595.1 \mathrm{~nm}$. 
electron transfer reaction. The figure suggests that in some configurations, sufficiently short distances can be achieved between donor and acceptor to allow an electron transfer through space : by ionisation of the donor, ejection and electron trapping by the acceptor moiety. Nevertheless, as in our system the electron donor and the electron acceptor are grafted to the same polymer chain, a transfer through bonds can not be excluded.

The triplet state of ZnTPP is assumed to be an intermediate state of the reaction. The assumption is supported by the fact that the lifetime of ZnTPP triplet is $26 \mathrm{~ms}^{22}$ and no persistent spectral hole could be burnt when the system was irradiated both by a $\lambda_{1}$ and $\lambda_{2}$ excitation with a time delay of $1 \mathrm{~s} .{ }^{21}$ In addition, the triplet has a high absorption cross-section at the gating excitation wavelength.

To explain the stability and the efficiency of photon gated holes in PMMA, some authors have considered a possible dissociative halogen detachement of the acceptor occurring after the electron transfer took place. This reaction was assumed to take place in TZT/halomethane/PMMA(TZT = meso-tetra-(p-tolyl)-Zn-tetrabenzoporphyrin $^{9,25}$ and TPP/AX/PMMA or polyethylene systems to explain the persistence of spectral holes (AX represents an acceptor molecule containing an halogen X). ${ }^{26}$ A similar mechanism could also be suspected in the case of our ZnTPP-PBCS/PMMA and ZnTPP-PBCS/PS systems.

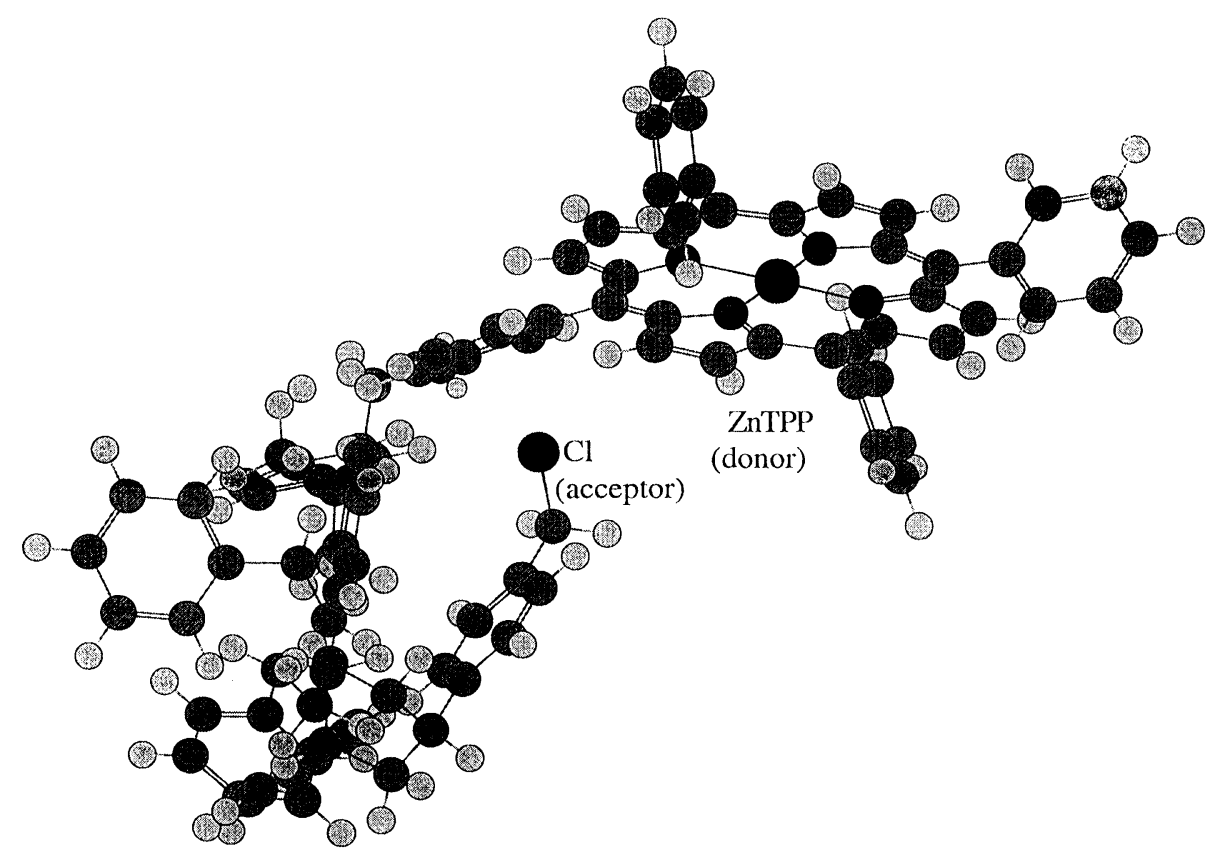

Polystyrene chain

Figure 6 A possible conformation of a fragment of the copolymer ZnTPP-PBCS chain showing the possibility of quite short distances between ZnTPP (donor) and benzylchloride (acceptor) moieties. 


\section{CONCLUSION}

In order to investigate spectral hole-burning, we have synthesised a co-polymer in which the ZnTPP as donor and vinylbenzylchloride as acceptor are linked in the same polystyrene chain. When the co-polymer is incorporated into PMMA or PS matrices, photon-gated holes are observed, with a higher efficiency in the PMMA host. The increase of the efficiency of hole formation under two-colour excitation instead of one-colour excitation achieve a factor 4 for ZnTPP-PBCS/PMMA. In contrast, no photon-gated hole-burning occurred when ZnTPP was dispersed in those matrices without acceptor, or in PS even with efficient electron acceptors. We believe that the spectral holes are produced by an electron transfer reaction from a high excited triplet state of ZnTPP to chlorine atoms in a benzylchloride group, closely located to the selectively excited ZnTPP grafted molecule. Our results obtained with ZnTPP-PBCS shows that systems with optimised donor and acceptor moieties grafted to a same polymer chain, should offer good prospects of D-A electron transfer systems for PSHB optical memory materials. The importance of host properties is also strongly underlined.

\section{Acknowledgements}

We are pleased to thank Professor Jean FAURE for supporting one of us (S. S.), for his invitation to S. K. for a post-doc stay in our laboratory, and for his critical reading of our manuscript.

\section{References}

1. W. E. Moerner, ed., Persistent Spectral Hole-burning, Sciences and Applications (Springer-Verlag, Berlin, 1988).

2. W. E. Moerner and M. D. Levenson, J. Opt. Soc. Am., B2 915 (1985).

3. R. M. Macfarlane, J. Lumin., 3820 (1987).

4. H. W. Lee, M. Gehrtz, E. E. Marinero and W. E. Moerner, Chem. Phys. Lett., 118611 (1985).

5. E. I. Alshits, B. M. Kharlamov and R. I. Personov, Opt. Spectrosc., 65326 (1988).

6. D. M. Burland and D. Haarer, IBM J. Res. Dev., 23534 (1979).

7. M. Iannone, G. W. Scott, D. Brinza and D. R. Coulter, J. Chem. Phys., 854863 (1986).

8. B. Prass, C. von Borczyskowski and D. Stehlik, J. Lumin, 3848 (1987).

9. T. P. Carter, C. Bräuchle, V. Y. Lee, M. Manavi and W. E. Moerner, J. Phys. Chem., 913998 (1987).

10. W. P. Ambrose and W. E. Moerner, Chem. Phys., 14471 (1990).

11. S. Machida, K. Horie and T. Yamashita, Appl. Phys. Lett., 60286 (1992).

12. S. Machida, K. Horie and T. Yamashita, J. Lumin., 5685 (1993).

13. H. Suzuki, T. Nishi, T. Shimada and H. Hiratsuka, J. Appl. Phys., 73374 (1993).

14. H. Suzuki, T. Shimada and H. Hiratsuka, Chem. Phys. Lett., 183570 (1991).

15. W. E. Moerner, Jpn. J. Appl. Phys. Suppl., 28221 (1989).

16. S. Salhi, M. C. Vernières, C. Bied-Charreton, J. Faure and A. Revillon, New J. Chem., 18783 (1994).

17. A. Harriman, G. Porter and A. Wilowska, J. Chem. Soc. Faraday Trans., 79-2 807 (1983).

18. A. Weller, Pure Appl. Chem., 16115 (1968).

19. D. Rehm and A. Weller, Isr. J. Chem., 8259 (1970).

20. M. Julliard, Photoinduced electron transfer, Part B. Experimental techniques and medium effects, eds. M. A. Fox and M. Chanon, (Elsevier, Amsterdam, 1988) p. 216.

21. H. Suzuki, T. Shimada, T. Nishi and H. Hiratsuka, Jpn. J. Appl. Phys., 29 L 1146 (1990).

22. J. R. Darwent, P. Douglas, A. Harriman, G. Porter and M. C. Richoux, Coord. Chem. Rev., 4483 (1982). 
23. C. K. Mann and K. K. Barnes, Electrochemical reactions in nonaqueous systems (Marcel Dekker, New-York, 1970) p.226.

24. B. Luo, M. Tian, W. Li, S. Hang and J. Yu, J. Lumin., 53247 (1992).

25. T. P. Carter, C. Bräuchle, V. Y. Lee, M. Manavi and W. E. Moerner, Opt. Lett., 12370 (1987).

26. H. Suzuki, T. Shimada and H. Hiratsuka, J. Appl. Phys., 704671 (1991). 